\title{
Effect of Localized Promotion of Cytokinin Biosynthesis on Flower Morphology in Flower Buds of Torenia fournieri Lind.
}

\author{
Tomoya Niki ${ }^{1,2}$, Ryutaro Aida ${ }^{1}$, Tomoko Niki ${ }^{1}$ and Takaaki Nishijima ${ }^{1,2 *}$ \\ ${ }^{1}$ NARO Institute of Floricultural Science, Tsukuba 305-8519, Japan \\ ${ }^{2}$ Graduate School of Life and Environmental Sciences, Tsukuba University, Tsukuba 305-8577, Japan
}

To analyze the relationship between flower morphology and organ-specific promotion of cytokinin biosynthesis within flower buds, we introduced Arabidopsis isopentenyltransferase 4 (AtIPT4) into torenia (Torenia fournieri L.) under the control of $A P E T A L A 1$ (AP1) or APETALA3 (AP3) promoter. AP1::AtIPT4 plants had an increased number of petals, whereas $A P 3:$ :AtIPT4 plants had an expanded corolla, a paracorolla, and serrated petal margins along with an increased number of petals. In $A P 3:$ :AtIPT4 plants, marked receptacle enlargement was observed when the flower buds were in the early corolla development stage in which the paracorolla primordia differentiate. As expected, AtIPT4 was expressed in the sepals and petals of AP1::AtIPT4 plants, and in the petals and stamens of AP3::AtIPT4 plants. Furthermore, the type-A response regulator (TfRR1) and cytokinin oxidase (TfCKX5) genes, which were used as indices of cytokinin signal, showed the same expression patterns as the transgene. These findings indicate that expansion of the corolla and development of the paracorolla and serrated petal margins after receptacle enlargement in $A P 3:: A t I P T 4$ plants are induced by localized elevated cytokinin signal in the petals and stamens. In contrast, localized elevated cytokinin signal in the sepals and petals only induced an increase in the number of petals. Therefore, an elevated cytokinin signal in the stamen may be important for inducing corolla expansion and for developing a paracorolla and serrated petal margins.

Key Words: flower morphology, genetic modification, isopentenyltransferase, paracorolla, spatial expression.

\section{Introduction}

Flower morphology is one of the most important commercial characteristics of floricultural plants. Flower morphologies such as double flowers, flowers with paracorollas and large flowers are attractive to consumers and are of great commercial value. Breeding programs to produce novel flower morphologies have mainly been based on mutant breeding, but this can be time consuming because the occurrence of desirable stable mutants is rare (Nishijima, 2007). Therefore, elucidation of the molecular mechanism underlying flower morphology may help produce a novel, more efficient breeding method by employing DNA markers and

Received; February 25, 2013. Accepted; July 4, 2013.

This study was supported by the Japan Society for the Promotion of Science (Grant-in-Aid KAKENHI No. 22580045) and the National Agriculture and Food Research Organization (Development of Innovative Crops through the Molecular Analysis of Useful Genes). Parts of this paper were presented at the 2009 autumn meeting of the Japanese Society for Horticultural Science.

* Corresponding author (E-mail: takaaki@affrc.go.jp). genetic transformation.

Cytokinin reportedly plays an important role in the regulation of flower morphology. In Arabidopsis, the number of floral organs, such as petals, stamens, and pistils, is increased after $N^{6}$-benzylaminopurine treatment of flower buds (Lindsay et al., 2006; Venglat and Sawhney, 1996). Such an increase in the number of floral organs is promoted by cytokinin-mediated induction of gene expression involved in meristematic activity and organ differentiation (Lindsay et al., 2006; Rupp et al., 1999). In Petunia hybrida, treatment with forchlorfenuron (CPPU), which inhibits cytokinin degradation through cytokinin oxidase/dehydrogenase (CKX) inhibition (Bilyeu et al., 2001), induces corolla expansion following an increase in the cell number (Nishijima, 2012; Nishijima et al., 2006). Furthermore, some genes involved in cytokinin biosynthesis and early signaling pathways are regulated by the Grandiflora gene, which is responsible for the large-flowered phenotype in petunia (Nishijima, 2012; Nishijima et al., 2011a, b).

In torenia, CPPU treatment induces several different flower morphologies, including an increase in the 
number of petals and development of the paracorolla and serrated petal margins (Nishijima and Shima, 2006). Of these flower morphologies, paracorollas are only observed in a few plant species, including Narcissus, Asclepias, Antirrhinum majus, and Passiflora, and contribute to the unique appearance of flowers. In addition, serrated petal margins, which are preferred as a 'fringed flower' type, also contribute to the increased diversity of flower morphologies. Varieties of new flowers could be produced if these flower morphologies could be universally induced in other floricultural plants.

The CPPU-induced changes in flower morphologies of torenia are dependent on the floral stage at which CPPU is applied; the petal-like wide paracorolla, which consists of wide extra petaloid organs, is induced when CPPU is applied at the sepal development stage (Stage 3 ), whereas the narrow paracorolla, which consists of narrow extra petaloid organs, is induced when CPPU is applied at the early corolla development stage (Stage 5). Serrated petal margins are induced when CPPU is applied at the middle corolla development stage (Stage 6; Nishijima and Shima, 2006). These morphological changes are induced by localized elevation of the cytokinin signal in flower buds (Niki et al., 2013).

Distribution of the cytokinin signal can be analyzed by monitoring the expression of cytokinin-responsive genes, i.e., type-A response regulator $(R R)$ and $C K X$ genes. The expressions of type-A $R R$ and $C K X$ genes are induced by cytokinin application and by an enhanced cytokinin signal (Brandstatter and Kieber, 1998; Brugière et al., 2003; D'Agostino et al., 2000; Kiba et al., 2005; Nishijima et al., 2011a, b; Taniguchi et al., 1998). Thus, expression of these genes reflects the extent of the cytokinin signal. In torenia, when CPPU application at Stage 3 induces wide paracorollas, the cytokinin signal is elevated on the abaxial side of the stamen primordia, which is the site of wide paracorolla initiation. When CPPU application at Stage 5 induces narrow paracorollas, the cytokinin signal is elevated in the middle part of young petals, which is the site of narrow paracorolla initiation. In contrast, the enhanced cytokinin signal by CPPU application at Stage 6 is localized from the middle to the apical parts of the petals when serrated petals are induced (Niki et al., 2013). Therefore, spatial distribution of the elevated cytokinin signal differs depending on the floral stage at CPPU application, which causes the induction of specific flower morphology. Further, morphological changes occur at the site of elevation of the cytokinin signal.

It is difficult to intentionally produce a particular flower morphology using conventional mutant breeding strategies. Utilization of plant growth regulators is also difficult because the chemical has to be precisely applied at a specific floral stage to obtain the desired uniform flower morphology. Therefore, the production of genetically modified plants expressing a cytokinin biosynthesis gene under the control of a floral organ- specific promoter would be an effective tool for more efficient breeding programs.

The biosynthetic pathways of cytokinins and their related genes in higher plants have been well documented (Frébort et al., 2011). The first step of cytokinin biosynthesis in higher plants is isopentenylation of ATP and ADP catalyzed by isopentenyltransferase (IPT) using dimethylallyl diphosphate as a substrate (Kakimoto, 2001; Takei et al., 2001). This is thought to be a ratelimiting step of cytokinin biosynthesis. In Arabidopsis, nine genes encoding IPT have been cloned, and transgenic plants overexpressing these genes show elevated concentrations of endogenous cytokinins (Sakakibara et al., 2005). Furthermore, the application of a promoter of floral homeotic genes would be useful for inducing transgenes in a floral organ-specific manner. The floral homeotic genes APETALA1 (API) and APETALA3 (AP3) are specifically expressed in whorls 1 and 2 and whorls 2 and 3, respectively (Jack et al., 1992; Mandel et al., 1992). Therefore, transgenic plants expressing IPT under the control of the promoters of these genes would specifically elevate cytokinin concentration and cytokinin signal in the sepals and petals and in the petals and stamens, respectively.

In this study, we introduced Arabidopsis IPT (AtIPT4) into torenia under the control of $A P 1$ or $A P 3$ promoters to clarify the effects of floral organ-specific promotion of cytokinin biosynthesis on flower morphology. On the basis of our results, a strategy to produce decorative flower morphologies is discussed.

\section{Materials and Methods}

\section{Plasmid construction and transformation of torenia}

For cloning AtIPT4 in Arabidopsis thaliana (AB061402), total RNA was isolated from flower buds and young flowers (Miyawaki et al., 2004) using an RNeasy Plant Mini Kit (Qiagen Sciences, Germantown, MD, USA) and RNase-Free DNase Set (Qiagen), and cDNA was synthesized using a Transcriptor First Strand cDNA Synthesis Kit (Roche, Mannheim, Germany), as described previously (Niki et al., 2012). For cloning Arabidopsis AP1 (At1g69120) and AP3 (At3g54340) promoters, genomic DNA was isolated from young Arabidopsis leaves using ISOPLANT II (Wako Pure Chemical Industries Ltd., Osaka, Japan). The open reading frame of AtIPT4 and the 5'-upstream region of Arabidopsis $A P 1$ and $A P 3$ were amplified using PCR with KOD Plus DNA polymerase (Toyobo, Osaka, Japan) with the following primers including an Eco RI site (underlined): forward 5'-CTCAGAATTCGACATG AAGTGT-3' and reverse 5'-CTAGTTAAGACTTAAAA ATCT-3' for AtIPT4 (970 bp); forward 5'-TGTATCGTT TCAAAACTCAGG-3' and reverse 5'-TACTGAATTC GAACCAAACAAAAC-3' for $A P 1$ (1729 bp); and forward 5'-GACCAGATCAAGAGTGCGTG-3' and reverse 5'-GTTTGAATTCTTTGTTGAAG-3' for $A P 3$ (1197 bp). All PCR fragments were digested using Eco 
RI; subsequently, the AtIPT4 fragment was ligated with the $A P 1$ or $A P 3$ fragment. The fragments produced were amplified by PCR using the following forward and reverse primers for AtIPT4 described above: forward 5'CACCTGTATCGTTTCAAAACTC-3' for AP $1::$ AtIPT4 (2689 bp), and forward 5'-CACCGACCAGATCAAGA GTGC-3' for AP3::AtIPT4 (2157 bp). These fragments were cloned into a $\mathrm{pENTR/D-TOPO}$ vector (Invitrogen, Carlsbad, CA, USA) and then transferred into a destination vector pGWB1 (Nakagawa et al., 2007), which was derived from the plant transformation vector pBI101, using the Gateway LR clonase reaction (Invitrogen).

Torenia fournieri 'Crown Violet' was used for transformation (Aida et al., 2000). Plant materials were vegetatively reproduced by herbaceous cuttings and grown at $25^{\circ} \mathrm{C}$ with illumination from fluorescent lamps ( $85 \mu \mathrm{mol} \cdot \mathrm{m}^{-2} \cdot \mathrm{s}^{-1}$ PPFD; $16 \mathrm{~h}$ light $/ 8 \mathrm{~h}$ dark cycle). The vectors were introduced into torenia by Agrobacteriummediated transformation as described by Aida and Shibata $(1995,2001)$. After selection with kanamycin and hygromycin, transformants were planted in horticultural soil (Kureha-Engei-Baido; Kureha Chemical Industry Co., Ltd., Tokyo, Japan) in plastic pots and grown in a greenhouse under natural light at $25^{\circ} \mathrm{C} / 20^{\circ} \mathrm{C}$ (day/night). Plants showing any changes in flower morphology were selected, and their $\mathrm{T}_{2}$ generations were obtained by selfing; the plants were used for subsequent investigations. Seeds of transgenic plants were germinated in another horticultural soil (Metro-Mix 350; Sun Gro Horticulture Canada Ltd, British Columbia, Canada), and the seedlings were transplanted into the horticultural soil in plastic pots and grown in the greenhouse as described above.

\section{Morphological analysis of flower bud development}

For observation of the morphological changes that occurred in the developing flower buds, flower buds at the sepal development stage (Stage 3), the stamen and pistil initiation stage (Stage 4), the early corolla development stage (Stage 5), and the middle corolla development stage (Stage 6), which are defined by Nishijima and Shima (2006), were fixed in FAA [50\% $(\mathrm{v} / \mathrm{v})$ ethanol, $10 \%(\mathrm{v} / \mathrm{v})$ formaldehyde, $5 \%(\mathrm{v} / \mathrm{v})$ acetic acid] at $4{ }^{\circ} \mathrm{C}$ overnight, then dehydrated through a graded ethanol series $[50 \%, 70 \%, 90 \%$, and $99.5 \%(\mathrm{v} / \mathrm{v})]$. The ethanol was replaced with 2-methyl-2-propanol. The samples were freeze-dried in 2-methyl-2-propanol and analyzed by scanning electron microscopy (SEM; VE7800; Keyence, Osaka, Japan).

\section{Quantitative real-time PCR analysis}

Total RNA was separately isolated from the sepals, petals, stamens, and pistils of the flower buds at Stage 6 from transgenic and non-transgenic plants using the same procedure used for cDNA cloning. Gene-specific primers for AtIPT4, torenia TfRR1 (AB740033), TfCKX5
(AB740039), and an actin gene (TfACT3; AB330989), which was used as an internal standard, were designed for the 3 '-terminal regions of the open reading frame and the 3'-untranslated regions of each gene. Primer sequences and the lengths of the PCR products used for quantitative real-time PCR reactions of AtIPT4 were forward 5'-ACAGCATCGTTTCGAGAGG-3' and reverse 5'-GTGGCTCCTGACAATCTTCAC-3' and $120 \mathrm{bp}$, respectively. The primers for TfRR1, TfCKX5, and TfACT3 were the same as those described previously (Niki et al., 2013). Gene expression was quantified using SYBR Premix Ex Taq (Takara Bio, Shiga, Japan) and quantitative real-time PCR (LightCycler; Roche). The PCR reactions were performed with an initial denaturation step of $10 \mathrm{~s}$ at $95^{\circ} \mathrm{C}$ followed by 50 cycles of $5 \mathrm{~s}$ at $95^{\circ} \mathrm{C}, 10 \mathrm{~s}$ at $60^{\circ} \mathrm{C}$, and $5-7 \mathrm{~s}$ at $72^{\circ} \mathrm{C}$. To avoid calculating non-specific $\mathrm{PCR}$ products, fluorescence was measured at the end of the extension phase at $76^{\circ} \mathrm{C}$ for $T f R R 1$, at $77^{\circ} \mathrm{C}$ for $T f A C T 3$, at $78^{\circ} \mathrm{C}$ for $T f C K X 5$, and at $80^{\circ} \mathrm{C}$ for AtIPT4. Raw data were analyzed with LightCycler software version 3.5 (Roche). Plasmids harboring full-length cDNA of the AtIPT4, TfRR1, or $T f C K X 5$ or a partial cDNA fragment of TfACT3 were used to obtain standard curves. The ratio of the expression of each gene to that of TfACT3 was calculated. Expression analyses were conducted independently and in triplicate.

\section{Analysis of endogenous cytokinins}

Endogenous cytokinins were extracted separately from the sepals, petals, stamens, and pistils at the late corolla development stage (Stage 7) from transgenic and non-transgenic plants. The methods used for cytokinin analysis were the same as those described by Dobrev and Kaminek (2002) and Nishijima et al. (2011a). Each floral organ $(0.2-0.6 \mathrm{~g} \mathrm{FW})$ was homogenized in liquid nitrogen and extracted in ice-cold $\mathrm{MeOH} /$ water/formic acid $(15 / 4 / 1, \mathrm{v} / \mathrm{v} / \mathrm{v})$, which includes $1 \mathrm{ng}$ of each internal standard, $\left[{ }^{2} \mathrm{H}_{6}\right] N^{6}-\left(\Delta^{2}\right.$-isopentenyl)adenine (iP), $\left[{ }^{2} \mathrm{H}_{5}\right]$ trans-zeatin (tZ), $\left[{ }^{2} \mathrm{H}_{6}\right]$ iP riboside (iPR), and $\left[{ }^{2} \mathrm{H}_{5}\right] \mathrm{tZ}$ riboside (tZR) (OlChemim, Olomouc, Czech Republic). After overnight extraction at $-20^{\circ} \mathrm{C}$, solids were separated using centrifugation. The extract was passed through a Sep-Pak $\mathrm{tC}_{18}$ cartridge (Waters, Milford, MA, USA) and evaporated to near dryness. The residue was dissolved in $1 \mathrm{M}$ formic acid and applied to an Oasis MCX column (Waters). The column was sequentially eluted with $\mathrm{MeOH}, 0.35 \mathrm{M} \mathrm{NH}_{4} \mathrm{OH}$, and $0.35 \mathrm{M} \mathrm{NH}_{4} \mathrm{OH}$ in $60 \%(\mathrm{v} / \mathrm{v})$ aqueous $\mathrm{MeOH}$. The eluent with $0.35 \mathrm{M}$ $\mathrm{NH}_{4} \mathrm{OH}$ in $60 \%(\mathrm{v} / \mathrm{v})$ aqueous $\mathrm{MeOH}$ comprising cytokinin nucleobases, cytokinin nucleosides, and cytokinin glucosides was evaporated to dryness. The dried sample was then dissolved in $10 \%$ aqueous $\mathrm{MeOH}$ containing $0.05 \%$ acetic acid and analyzed using a liquid chromatography-tandem mass spectrometry system (LC/MS/MS, model 2695/TSQ7000; Waters/Thermo Fisher Scientific, Waltham, MA, USA) coupled with 
positive ion electrospray ionization. Cytokinins were separated using an ODS column (MD, $5 \mu \mathrm{m}, 2.5 \mathrm{~mm} \times$ $250 \mathrm{~mm}$; Shiseido Fine Chemicals, Tokyo, Japan) at a flow rate of $0.2 \mathrm{~mL} \cdot \mathrm{min}^{-1}$ with gradients of solvents A ( $\mathrm{MeOH}$ containing $0.05 \%$ acetic acid) and $\mathrm{B}$ (water containing $0.05 \%$ acetic acid) as follows: $0 \mathrm{~min}, 10 \% \mathrm{~A}$ $+90 \% \mathrm{~B} ; 45 \mathrm{~min}, 80 \% \mathrm{~A}+20 \% \mathrm{~B}$; and $55 \mathrm{~min}, 100 \%$ A. The column temperature was $35^{\circ} \mathrm{C}$. Quantification was performed in selected ion recording mode. The ionization voltage was $4.7 \mathrm{kV}$, the capillary temperature was $200^{\circ} \mathrm{C}$, and the collision energy was -22 to $-34 \mathrm{~V}$ depending on the cytokinin species. Data were analyzed using Xcaliber software (Thermo Fisher Scientific). Endogenous cytokinins were quantified by the internal standard method using the corresponding deuterated cytokinins. Quantification of endogenous cytokinins was conducted independently and in triplicate.

\section{Results and Discussion}

Changes to the flower morphology of transgenic torenia Several morphological changes were induced in the flowers of both API::AtIPT4 and AP3::AtIPT4 plants, and these traits were inherited. The number of petals in AP1::AtIPT4 plants was increased to 6-7 (Fig. 1b) from the characteristic 5 petals of the normal type (NT) plants (Fig. 1a). The number of sepals was increased to 5-7 in AP $1::$ AtIPT4 plants, whereas the NT plants had 5 sepals. In contrast, the number of stamens and pistils remained unchanged. In AP3::AtIPT4 plants, the number of petals increased to 5-6 (Fig. 1c, d), although the increase was moderate compared with $A P 1:: A t I P T 4$ plants (Fig. 1b). In addition, marked petal expansion and serrated petal margins were observed in AP3::AtIPT4 plants (Fig. 1c, d), but not in NT plants (Fig. 1a). Furthermore, extra floral organs resembling petals were observed on the corolla (Fig. 1d). AP3::AtIPT4 plants had 4-5 stamens compared with the 4 stamens of NT plants, but the number of sepals and pistils was unchanged.

In Stage 3 of floral development, both NT and AP3::AtIPT4 plants had 5 sepals (Fig. 2a, o); however, $A P 1:: A t I P T 4$ plants had more than 6 sepals (Fig. 2h). In the initiation stage of the stamen and pistil (Stage 4),
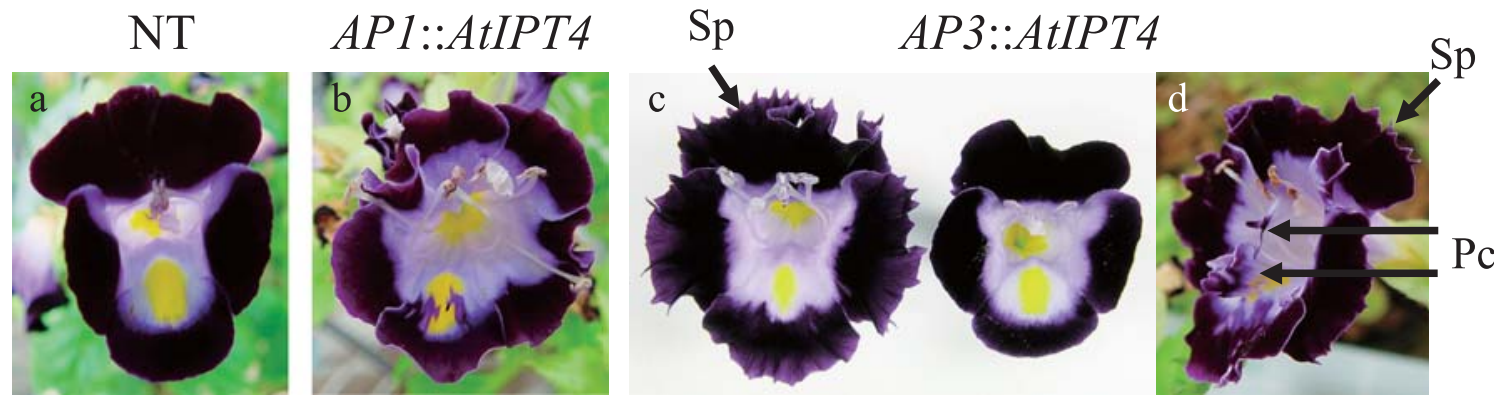

Fig. 1. Morphological changes in the flowers of torenia. a, Normal type (NT); b, AP1::AtIPT4; c and d, AP3::AtIPT4. Pc, Paracorolla; Sp, Serrated petal.
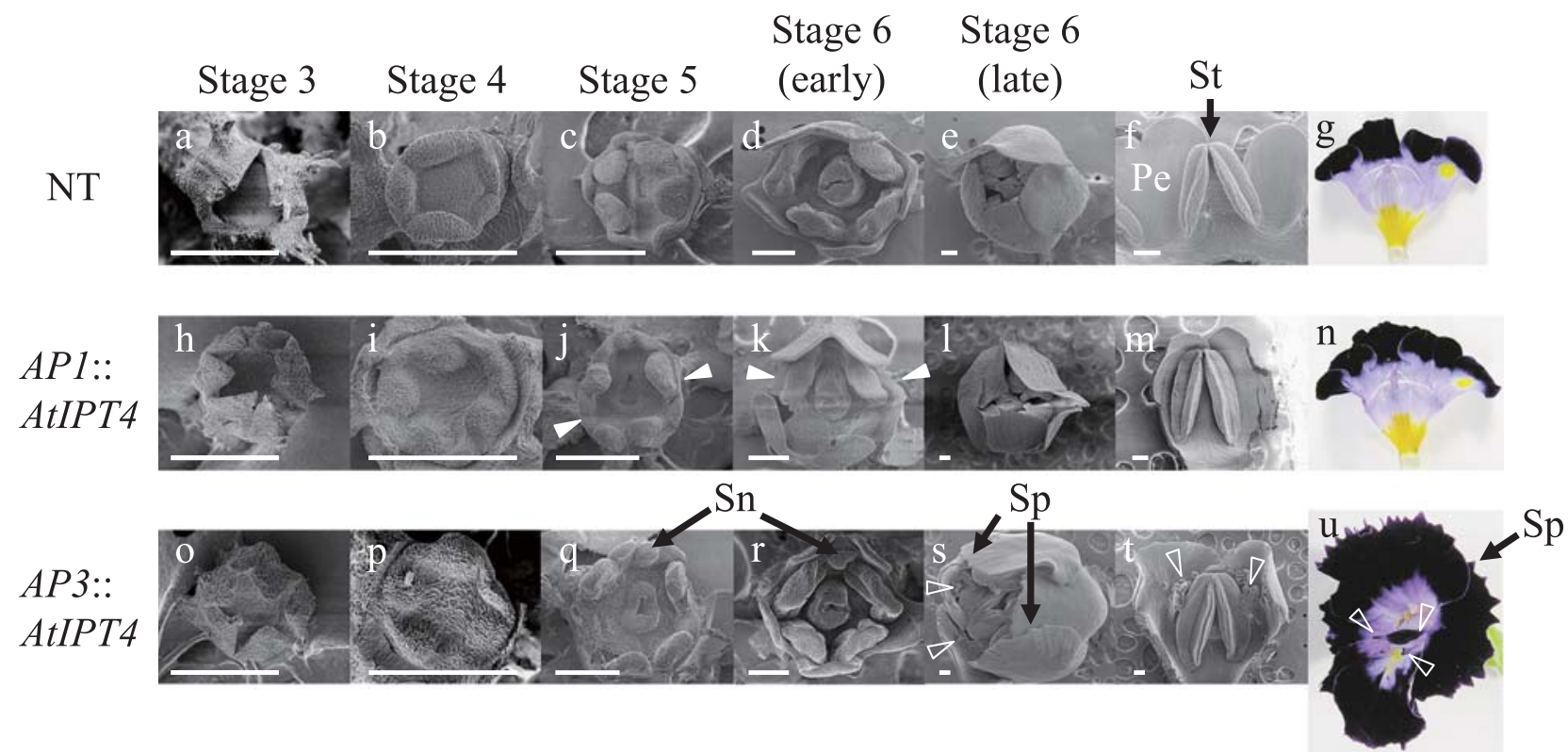

Fig. 2. Floral development of transgenic torenia. a-g, Normal type (NT); h-n, AP $1::$ AtIPT4; o-u, AP3::AtIPT4. Floral stages were defined as described by Nishijima and Shima (2006). Closed and open triangles represent lobes from a petal primordium and the site of paracorolla initiation, respectively. Pe, Petal; Sn, Staminoid; Sp, Serrated petal; St, Stamen. Scale bars $=100 \mu \mathrm{m}$. 
the receptacle was larger in both AP1::AtIPT4 and AP3::AtIPT4 plants (Fig.2i, p) than in NT plants (Fig. 2b). Furthermore, the receptacle of AP3::AtIPT4 plants became markedly larger than those of NT and AP1::AtIPT4 plants at Stage 5 (Fig. 2c, j, q). In Stage 5 and early Stage 6 , an increase in the number of petal primordia, was observed in $A P 1:: A t I P T 4$ plants (Fig. 2c, $\mathrm{d}, \mathrm{g}, \mathrm{j}, \mathrm{k}, \mathrm{n})$. The staminoid of AP3::AtIPT4 plants was also larger than that of the NT and AP1::AtIPT4 plants (Fig. 2c, d, j, k, q, r). In late Stage 6, primordia of the extra floral organs were initiated and corolla expansion and serrated petal margins were observed in AP3::AtIPT4 plants (Fig. 2s). These extra floral organs were formed on the abaxial and lateral sides of the basal part of the stamen, which corresponded to the middle part of the petal (Fig. 2t, u). The morphology of the extra floral organs resembled the narrow paracorollas induced by CPPU treatment (Niki et al., 2012; Nishijima and Shima, 2006; Figs. 1d and $2 \mathrm{u}$ ).

The paracorolla originates from the stipule of the stamen in Narcissus, Asclepias, and A. majus, and from the receptacle in Passiflora (Troll, 1957; Yamaguchi et al., 2010). The site of paracorolla differentiation depends on its origin. Paracorolla primordia differentiate on the abaxial and lateral sides of the stamen primordia when the paracorolla originates from the stipule of the stamen in A.majus (Yamaguchi et al., 2010) and the CPPUtreated torenia flowers (Nishijima and Shima, 2006). In AP3::AtIPT4 plants of torenia, the site and the floral stage in which the extra floral organ initiation occurred coincided with those of the narrow paracorollas induced by CPPU treatment and those of the A. majus paracorolla (Fig. $2 \mathrm{~s}, \mathrm{t}$ ). Therefore, together with the morphological similarity to the narrow paracorollas as described above, the extra floral organs formed in AP3::AtIPT4 flowers may be paracorollas originating from the stipule of the stamen.

\section{Cytokinin biosynthesis and signal transduction in transgenic torenia}

As expected, the AtIPT4 transgene was mainly expressed in the sepals and petals of AP1::AtIPT4 plants and in the petals and stamens of AP3::AtIPT4 plants (Fig. 3).

In NT plants, iP and iPR were not detected in the sepals and petals, while the stamens contained high concentrations of iP (Fig. 4a, b). The concentrations of $\mathrm{iP}$ in the pistils and iPR in the stamens and pistils were slight in NT plants (Fig. 4a, b). Both iP and iPR accumulated in the sepals and petals in AP1::AtIPT4 plants, whereas changes in iP and iPR concentrations in the stamens and pistils compared with those of NT plants were obscure (Fig. 4a, b). On the other hand, both iP and iPR accumulated in the petals of AP3::AtIPT4 plants, but were undetected in the sepals, whereas changes in iP and iPR concentrations in the stamens and pistils compared with those of NT plants were obscure (Fig. 4a,

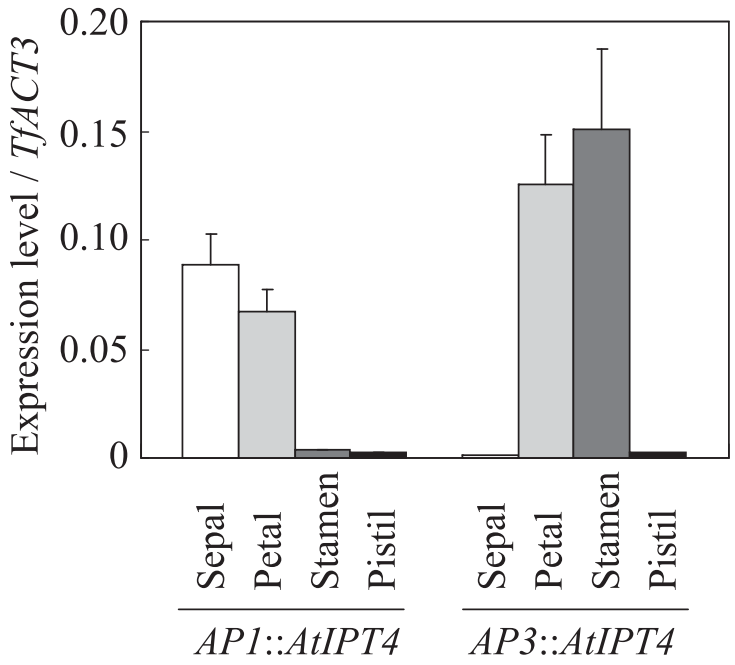

Fig. 3. AtIPT4 expression in the floral organs of transgenic torenia. The expression levels are shown as a value relative to that of TfACT3, which was used as an internal standard. Vertical bars represent $\pm \operatorname{SE}(\mathrm{n}=3)$.

b). The other cytokinin species quantified, tZ and tZR, were accumulated mainly in the stamen in NT plants, although they were also detected in the other floral organs (Fig. 4c, d). The accumulations of tZ and tZR among the floral organs in AP1::AtIPT4 and AP3::AtIPT4 plants showed mostly the same pattern as those of NT plants, except that tZR was higher in the sepals of AP1::AtIPT4 plants than in those of NT plants (Fig. 4c, d).

Because the changes of cytokinin concentrations in the stamens of AP3::AtIPT4 plants from those of NT plants were obscure, we analyzed the expressions of TfRR1 and TfCKX5, which reflect the extent of the cytokinin signal as described above. The expressions of $T f R R 1$ and $T f C K X 5$ were low or undetectable in all floral organs of NT plants (Fig. 5). In APl::AtIPT4 plants, the expressions of TfRR 1 and TfCKX5 in the sepals and petals were more than 10-fold that in NT plants (Fig. 5). On the other hand, the expressions of TfRRI and TfCKX5 were more than 10-fold higher in the petals and stamens of AP3::AtIPT4 than in NT plants (Fig. 5). These expression patterns among floral organs coincided with those of the introduced AtIPT4.

Why was endogenous cytokinin concentration in the stamens of AP3::AtIPT4 plants not markedly different from that in NT plants (Fig. 4a, b)? The stamen is the organ synthesizing cytokinins because cytokinin is necessary for pollen development (Huang et al., 2003; Sawhney and Shukla, 1994). At the same time, the development of the anther and pollen is inhibited by elevated cytokinin concentrations in transgenic tobacco plants overexpressing IPT in an anther-specific manner (Geng et al., 2002). This suggests that normal development of the stamen would be inhibited by excess cytokinin signal in the anther. Therefore, in the stamens of AP3::AtIPT4 torenia plants, intensive expression of AtIPT4 possibly had an inhibitory effect on stamen 

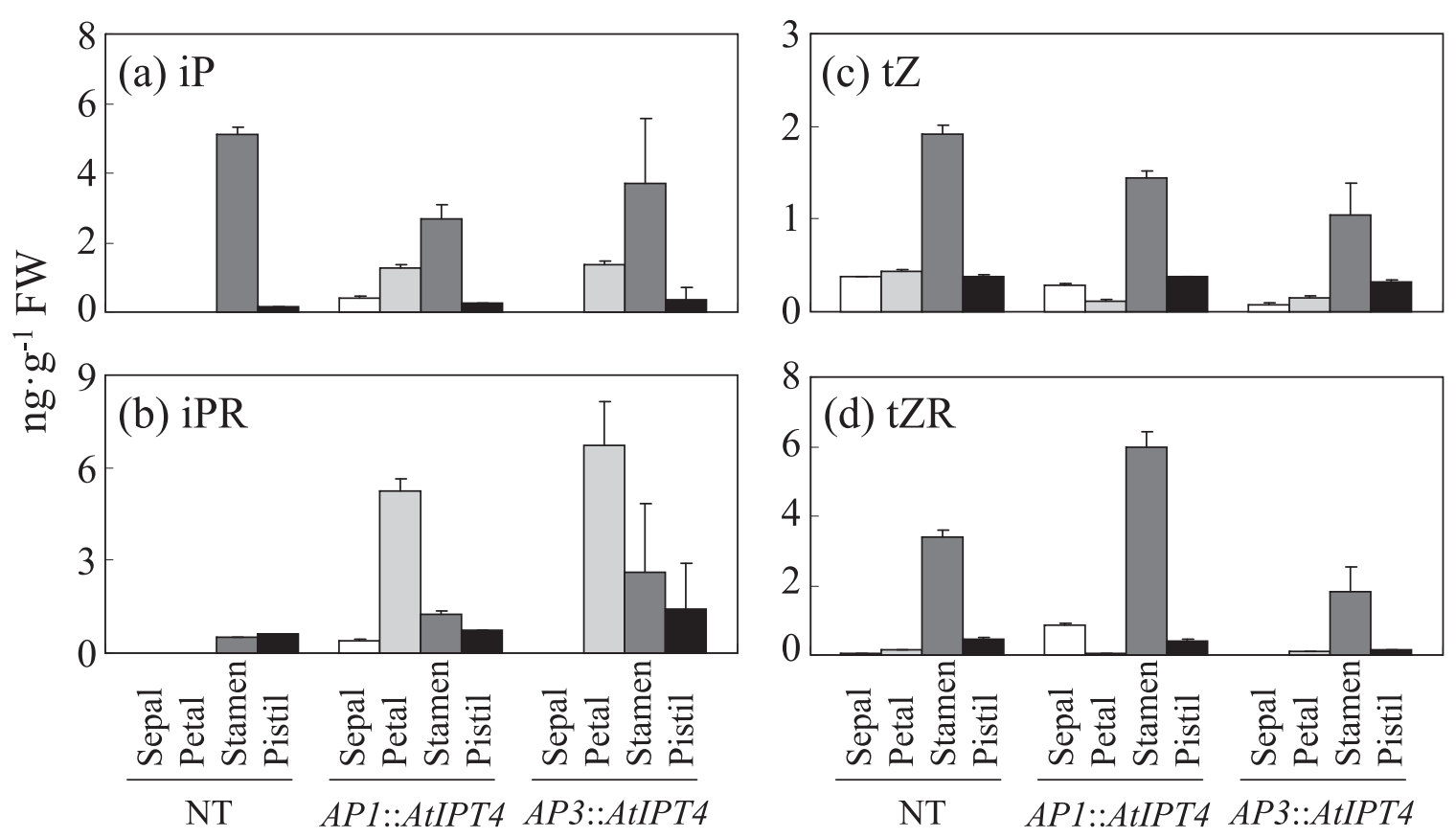

Fig. 4. Concentration of endogenous cytokinins in the floral organs of normal type (NT) and transgenic torenia. iP, isopentenyladenine; iPR, isopentenyladenine riboside; $\mathrm{tZ}$, trans-zeatin; tZR, trans-zeatin riboside. Vertical bars represent $\pm \mathrm{SE}(\mathrm{n}=3)$.

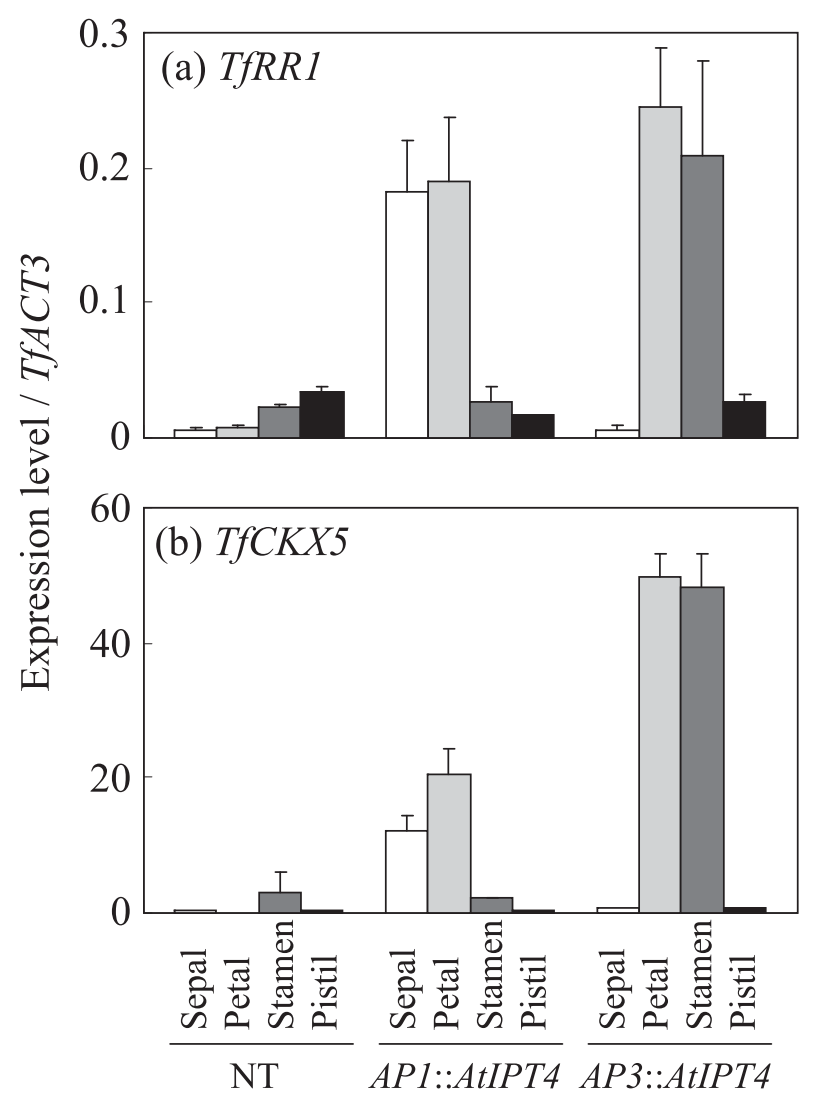

Fig. 5. Expression of $T f R R 1$ and $T f C K X 5$ in the floral organs of normal type (NT) and transgenic torenia. The expression levels are shown as values relative to that of TfACT3, which was used as an internal standard. Vertical bars represent $\pm \operatorname{SE}(n=3)$. development, which may have been a barrier to cytokinin biosynthesis.

Taken together, these results indicate that enhanced cytokinin signal in the sepals and petals of $A P 1:: A t I P T 4$ plants and in the petals and stamens of AP3::AtIPT4 plants induced flower morphologies specific to the transgenes.

In Arabidopsis, the number of sepals, petals, and stamens increases when AtIPT4 is expressed under the control of AP1 promoter (Li et al., 2010). Some morphological changes, such as the development of a small petal on top of the filament, a lobed petal, and the formation of the filament and a small petal between the sepal and petal, are observed in transgenic Arabidopsis overexpressing $I P T$ in an anther-specific manner (Geng et al., 2002). Furthermore, IPT expression under the control of $A P 3$ promoter leads to an increase in the size of the corolla in petunia (Verdonk et al., 2008). However, there has been no research analyzing the relationship between spatial distributions of elevated cytokinin and flower morphologies using the same plant material under the same experimental condition. In our study, the increase in petal number, corolla expansion, and the development of paracorollas and serrated petal margins were induced by localized expression of AtIPT4 in the petals and stamens of torenia plants, whereas only the petal number increased when AtIPT4 was expressed in the sepals and petals (Figs. 1 and 2). These results indicate that an elevated cytokinin signal in the stamen may be critical for corolla expansion, development of paracorollas and serrated petal margins (Fig. 6).

In Arabidopsis, the increase in the number of floral organs in response to the application of cytokinin has 


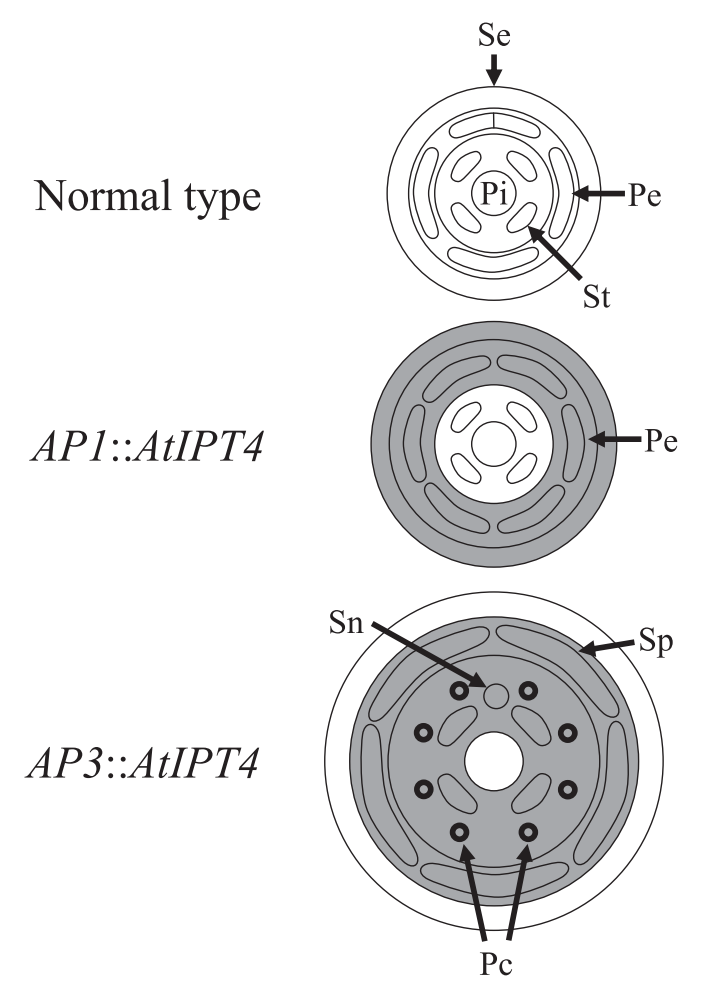

Fig. 6. Model of the effect of localized elevated cytokinin concentration in flower buds on flower morphology in transgenic torenia. Whorls with elevated cytokinin levels are represented in gray. Pc, Paracorolla; Pe, Petal; Pi, Pistil; Se, Sepal; Sn, Staminoid; Sp, Serrated petal; St, Stamen.

been shown to be caused by an enlarged floral meristem, and consequently by an enlarged receptacle (Bartrina et al., 2011; Lindsay et al., 2006). In addition, in Arabidopsis, an enlarged receptacle is observed in AP1::AtIPT4 plants with an increase in the number of floral organs (Li et al., 2010). Because the organ primordia tend to differentiate at constant intervals, the number of organs increases when the receptacle is enlarged. In our study, receptacle enlargement was observed at Stage 4 in both AP1::AtIPT4 and AP3::AtIPT4 torenia plants (Fig. 2i, p). This suggests that elevated cytokinin signal in whorl 2 causes receptacle enlargement, resulting in an increase in the number of petals (Fig. 6). However, only AP3::AtIPT4 plants showed enhanced receptacle progressively in Stage 5 (Fig. 2q). Enlargement preferably occurs in whorl 3 . When enhanced receptacle enlargement continues to Stage 5, the distance between each stamen is increased markedly (Fig. 6). Because the stipule of the stamen, which develops into paracorolla, is located on the lateral sides of the stamen and included in whorl 3, sufficient space allowing paracorolla primordia to be initiated may have been supplied. The same phenomenon is observed when the paracorollas are induced by CPPU treatment (Nishijima and Shima, 2006). That is, the paracorollas are generated synchronously with receptacle enlargement at Stage 5. In Arabidopsis, receptacle enlargement by cytokinin has been shown to be caused through coordinated regulation of WUSCHEL (WUS) and CLAVATA (CLV) (Bartrina et al., 2011; Clark et al., 1993; Lindsay et al., 2006). WUS, which maintains meristematic activity of the shoot apical meristem (SAM), expressed in the central zone in SAM. WUS expression is restricted in the central zone by $C L V 1$, which promotes organ differentiation (Schoof et al., 2000). Cytokinin expands the WUS expression area and consequently meristem size through repression of $C L V 1$ expression (Bartrina et al., 2011; Lindsay et al., 2006). This regulation mechanism of SAM size is thought to function also in the floral meristem, which regulates receptacle size (Bartrina et al., 2011; Lindsay et al., 2006). In our transgenic torenia, if $C L V$ expression was repressed at the site where the cytokinin signal was elevated, it could be presumed that elevation of the cytokinin signal in whorl 3 would be more effective for receptacle enlargement than that in whorl 2. This is because whorl 3 is located more closely to the central zone of the floral meristem than whorl 2. Meanwhile, it can't be ruled out that the elevated cytokinin signal in whorl 3 of AP3::AtIPT4 torenia induced the development of the stipule of the stamen independent of any receptacle enlargement. This is because cytokinin promotes organ differentiation and development by directly promoting the meristematic activity of the organ (Dewitte et al., 1999; Mok and Mok, 2001; Pernisová et al., 2009).

Serrated petal margins have been shown to be generated by changes in the arrangement of the vascular bundle induced by an elevated cytokinin signal at the petal limb (Niki et al., 2013; Nishijima and Shima, 2006). In contrast, we demonstrated that an elevated cytokinin signal in the stamen was critical for inducing a serrated petal margin, as described above. A serrated petal margin is also induced in a transgenic torenia in which the function of AGAMOUS $(A G)$, a floral homeotic gene expressed in whorls 3 and 4, is inhibited by a plantspecific transcriptional repression domain ( $A G-S R D X$, Narumi et al., 2008). This shows that a functional change in a gene expressed in whorls 3 and 4 may affect the arrangement of the vascular bundle in whorl 2. The elevated cytokinin signal in whorl 3 , which inevitably alters the gene expression profile, remotely affects the vascular bundle arrangement in the petal.

In our study, corolla expansion and a serrated petal margin were stable phenotypes in transgenic torenia (Figs. 1 and 6), which suggests that an elevated cytokinin signal in the petals and stamens can be practically applied for the development of novel flower morphologies. However, the induction of the narrow paracorolla observed in AP3::AtIPT4 plants was unstable (Figs. 1d and $2 \mathrm{u}$ ). On the other hand, CPPU treatment induced a petal-like wide paracorolla, which makes a stronger impression than the narrow paracorolla (Niki et al., 2012; Nishijima and Shima, 2006; Figs. $1 \mathrm{~d}$ and 2u). The delicate regulation of spatial and temporal accumulation of the cytokinin signal is necessary to induce a wide 
paracorolla (Niki et al., 2013). Because the transgene was expressed in the whole area of whorls 2 and 3 by the AP3 promoter in this study, more precisely and intensively targeted cytokinin accumulation in floral organs may be necessary to induce a wide paracorolla. As described, the type of CPPU-induced flower morphology is highly dependent on the floral stage at which CPPU is applied (Nishijima and Shima, 2006); however, such floral stage-dependent regulation of cytokinin accumulation is impossible in the AP3::AtIPT4 construct, thereby making paracorolla induction unstable. Therefore, a floral stage-specific promoter may be needed to ensure the stable induction of paracorollas. In the future, novel breeding methods for producing particular flower morphologies may be established if new transgenic technologies enabling spatial and temporal localization of cytokinin signal in the manner described above are developed.

\section{Acknowledgements}

We thank Dr. Tsuyoshi Nakagawa for kindly supplying us with the pGWB1 vector. We also thank Mrs. Yoshiko Kashiwagi, Mrs. Tomoko Kurobe, Mrs. Satoko Ohtawa, and Mrs. Yasuko Taniji for their technical assistance.

\section{Literature Cited}

Aida, R. and M. Shibata. 1995. Agrobacterium-mediated transformation of torenia (Torenia fournieri). Breed. Sci. 45: $71-74$.

Aida, R. and M. Shibata. 2001. Transgenic Torenia fournieri Lind (torenia). p. 294-305. In: Y. P. S. Bajaj (ed.). Biotechnology in agriculture and forestry, vol 48 transgenic crops III. Springer, Berlin.

Aida, R., S. Kishimoto, Y. Tanaka and M. Shibata. 2000. Modification of flower color in torenia (Torenia fournieri Lind.) by genetic transformation. Plant Sci. 153: 33-42.

Bartrina, I., E. Otto, M. Strnad, T. Werner and T. Schmülling. 2011. Cytokinin regulates the activity of reproductive meristems, flower organ size, ovule formation, and, thus, seed yield in Arabidopsis thaliana. Plant Cell 23: 69-80.

Bilyeu, K. D., J. L. Cole, J. G. Laskey, W. R. Riekhof, T. J. Esparza, M. D. Kramer and R. O. Morris. 2001. Molecular and biochemical characterization of a cytokinin oxidase from maize. Plant Physiol. 125: 378-386.

Brandstatter, I. and J. J. Kieber. 1998. Two genes with similarity to bacterial response regulators are rapidly and specifically induced by cytokinin in Arabidopsis. Plant Cell 10: 10091019.

Brugière, N., S. Jiao, S. Hantke, C. Zinselmeier, J. A. Roessler, X. Niu, R. J. Jones and J. E. Habben. 2003. Cytokinin oxidase gene expression in maize is localized to the vasculature, and is induced by cytokinins, abscisic acid, and abiotic stress. Plant Physiol. 132: 1228-1240.

Clark, S. E., M. P. Running and E. M. Meyerowitz. 1993. CLAVATA1, a regulator of meristem and flower development in Arabidopsis. Development 119: 397-418.

D'Agostino, I. B., J. Deruère and J. J. Kieber. 2000. Characterization of the response of the Arabidopsis response regulator gene family to cytokinin. Plant Physiol. 124: 17061717.

Dewitte, W., A. Chiappetta, A. Azmi, E. Witters, M. Strnad, J.
Rembur, M. Noin, D. Chriqui and H. Van Onckelen. 1999. Dynamics of cytokinins in apical shoot meristems of a dayneutral tobacco during floral transition and flower formation. Plant Physiol. 119: 111-121.

Dobrev, P. I. and M. Kaminek. 2002. Fast and efficient separation of cytokinins from auxin and abscisic acid and their purification using mixed-mode solid phase extraction. J. Chromatogr. A 950: 21-29.

Frébort, I., M. Kowalska, T. Hluska, J. Frébortová and P. Galuszka. 2011. Evolution of cytokinin biosynthesis and degradation. J. Exp. Bot. 62: 2431-2452.

Geng, S., M. Ma, H-C. Ye and G-F. Li. 2002. Anther-specific expression of ipt gene in transgenic tobacco and its effect on plant development. Transgenic Res. 11: 269-278.

Huang, S,. R. E. Cerny, Y. Qi, D. Bhat, C. M. Aydt, D. D. Hanson, K. P. Malloy and L. A. Ness. 2003. Transgenic studies on the involvement of cytokinin and gibberellin in male development. Plant Physiol. 131: 1270-1282.

Jack, T., L. L. Brockman and E. M. Meyerowitz. 1992. The homeotic gene APETALA3 of Arabidopsis thaliana encodes a MADS box and is expressed in petals and stamens. Cell 68: 683-687.

Kakimoto, T. 2001. Identification of plant cytokinin biosynthetic enzymes as dimethylallyl diphosphate: ATP/ADP isopentenyltransferases. Plant Cell Physiol. 42: 677-685.

Kiba, T., T. Naitou, N. Koizumi, T. Yamashino, H. Sakakibara and T. Mizuno. 2005. Combinatorial microarray analysis revealing Arabidopsis genes implicates in cytokinin responses through the His $\rightarrow$ Asp phosphorelay circuitry. Plant Cell Physiol. 46: 339-355.

Li, X. G., Y. H. Su, X. Y. Zhao, W. Li, X. Q. Gao and X. S. Zhang. 2010. Cytokinin overproduction-caused alteration of flower development is partially mediated by $C U C 2$ and $C U C 3$ in Arabidopsis. Gene 450: 109-120.

Lindsay, D. L., V. K. Sawhney and P. C. Bonham-Smith. 2006. Cytokinin-induced changes in CLAVATA1 and WUSCHEL expression temporary coincide with altered floral development in Arabidopsis. Plant Sci. 170: 1111-1117.

Mandel, M. A., C. Gustafson-Brown, B. Savidge and M. F. Yanofsky. 1992. Molecular characterization of the Arabidopsis floral homeotic gene APETALA1. Nature 360: 273-277.

Miyawaki, K., M. Matsumoto-Kitano and T. Kakimoto. 2004. Expression of cytokinin biosynthetic isopentenyltransferase genes in Arabidopsis: tissue specificity and regulation by auxin, cytokinin, and nitrate. Plant J. 37: 128-138.

Mok, D. W. and M. C. Mok. 2001. Cytokinin metabolism and action. Annu. Rev. Plant Physiol. Plant Mol. Biol. 52: 89-118.

Nakagawa, T., T. Kurose, T. Hino, K. Tanaka, M. Kawamukai, Y. Niwa, K. Toyooka, K. Matsuoka, T. Jinbo and T. Kimura. 2007. Development of series of gateway binary vectors, pGWBs, for realizing efficient construction of fusion genes for plant transformation. J. Biosci. Bioeng. 104: 34-41.

Narumi, T., R. Aida, T. Niki, T. Nishijima, N. Mitsuda, K. Hiratsu, M. Ohme-Takagi and N. Ohtsubo. 2008. Chimeric AGAMOUS repressor induces serrated petal phenotype in Torenia fournieri similar to that induced by cytokinin application. Plant Biotechnol. 25: 45-53.

Niki, T., M. Hirai, T. Niki, A. Kanno and T. Nishijima. 2012. Role of floral homeotic genes in the morphology of forchlorfenuron-induced paracorollas in Torenia fournieri Lind. J. Japan. Soc. Hort. Sci. 81: 204-212.

Niki, T., T. Mahesumu, T. Niki and T. Nishijima. 2013. Localized high expression of type-A response regulator and cytokinin oxidase/dehydrogenase genes in relation to forchlorfenuroninduced changes in flower morphology in Torenia fournieri 
Lind. J. Japan. Soc. Hort. Sci. 82: 69-77.

Nishijima, T. 2007. Hanagata. p. 37-43. In: Nosangyosonbunkakyokai (ed.). Nogyogijututaikei Kakihen 5 (Tsuiroku) 9 (In Japanese). Nosangyosonbunkakyokai, Tokyo.

Nishijima, T. 2012. Large flower size: Molecular basis and role of cytokinin. J. Japan. Soc. Hort. Sci. 81: 129-139.

Nishijima, T. and K. Shima. 2006. Change in flower morphology of Torenia fournieri Lind. induced by forchlorfenuron application. Sci. Hortic. 109: 254-261.

Nishijima, T., H. Miyaki, K. Sasaki and T. Okazawa. 2006. Cultivar and anatomical analysis of corolla enlargement of petunia (Petunia hybrida Vilm.) by cytokinin application. Sci. Hortic. 111: 49-55.

Nishijima, T., T. Niki and T. Niki. 2011a. Corolla of the largeflowered petunia (Petunia hybrida Vilm.) cultivars exhibit low endogenous cytokinin concentration through enhanced expression of the genes encoding cytokinin oxidases. J. Japan. Soc. Hort. Sci. 80: 334-342.

Nishijima, T., T. Niki and T. Niki. 2011b. The large-flowered petunia (Petunia hybrida Vilm.) genotype promotes expressions of type-A response regulator and cytokinin receptor genes like cytokinin response. J. Japan. Soc. Hort. Sci. 80: 343-350.

Pernisová, M., P. Klíma, J. Horák, M. Válková, J. Malbeck, P. Soucek, P. Reichman, K. Hoyerová, J. Dubová, J. Friml, E. Za ímalová and J. Hejátko. 2009. Cytokinins modulate auxininduced organogenesis in plants via regulation of the auxin efflux. Proc. Natl. Acad. Sci. USA 106: 3609-3614.

Rupp, H. M., M. Frank, T. Werner, M. Strnad and T. Schmülling. 1999. Increased steady state mRNA levels of the STM and KNATI homeobox genes in cytokinin overproducing Arabidopsis thaliana indicate a role for cytokinins in the shoot apical meristem. Plant J. 18: 557-563.

Sakakibara, H., H. Kasahara, N. Ueda, M. Kojima, K. Takei, S. Hishiyama, T. Asami, K. Okada, Y. Kamiya, T. Yamaya and
S. Yamaguchi. 2005. Agrobacterium tumefaciens increases cytokinin production in plastids by modifying the biosynthetic pathway in the host plant. Proc. Natl. Acad. Sci. USA 102: 9972-9977.

Sawhney, V. K. and A. Shukla. 1994. Male sterility in flowering plants: Are plant growth substances involved? Am. J. Bot. 81: 1640-1647.

Schoof, H., M. Lenhard, A. Haecker, K. F. X. Mayer, G. Jürgens and T. Laux. 2000. The stem cell population of Arabidopsis shoot meristems is maintained by a regulatory loop between the CLAVATA and WUSCHEL genes. Cell 100: 635-644.

Takei, K., H. Sakakibara and T. Sugiyama. 2001. Identification of genes encoding adenylate isopentenyltransferase, a cytokinin biosynthesis enzyme, in Arabidopsis thaliana. J. Biol. Chem. 276: 26405-26410.

Taniguchi, M., T. Kiba, H. Sakakibara, C. Ueguchi, T. Mizuno and T. Sugiyama. 1998. Expression of Arabidopsis response regulator homologs is induced by cytokinins and nitrate. FEBS Lett. 429: 259-262.

Troll, W. 1957. Praktische Einführung in die Pflanzenmorphologie 2. Fischer, Jena. (Troll, W. 2004. Zusetu-shokubutukeitaigakuhandobukku 2 (In Japanese). Translated by S. Nakamura and H. Tobe. Asakurashoten, Tokyo.)

Venglat, S. P. and V. K. Sawhney. 1996. Benzylaminopurine induces phenocopies of floral meristem and organ identity mutants in wild-type Arabidopsis plants. Planta 198: 480-487.

Verdonk, J. C., K. Shibuya, H. M. Loucas, T. A. Colquhoun, B. A. Underwood and D. G. Clark. 2008. Flower-specific expression of the Agrobacterium tumefaciens isopentenyltransferase gene results in radial expansion of floral organs in Petunia hybrida. Plant Biotechnol. J. 6: 694-701.

Yamaguchi, H., T. Niki, T. Niki and T. Nishijima. 2010. Morphological property and role of homeotic genes in paracorolla development of Antirrhinum majus. J. Japan. Soc. Hort. Sci. 79: 192-199. 